\title{
Théâtre, émotion et mémoire. Des fragments d'intime au collectif
}

\author{
Entretien avec Didier Ruiz
}

\begin{abstract}
Metteur en scène et comédien, Didier Ruiz débute son projet sur la mémoire «Dale Recuerdos, Je pense à Vous » en 1999, à l'occasion d'une carte blanche qui lui a été octroyée dans la ville de Béziers. Depuis, vingt-deux différentes éditions se sont succédées, en France, mais aussi au Chili, en Espagne et en Russie où la pièce a été récompensée en 2009 d'un « Masque d'or », prix très prestigieux du théâtre russe.
\end{abstract}

Marina Repezza : Comment décririez-vous ce projet que vous portez sur scène avec des personnes âgées depuis 1999 ?

Didier Ruiz : Sur le plateau, il y a une rangée de chaises. L'entrée est collective, les participants, une dizaine en général, se placent sur la ligne de présentation dans un ordre établi et à tour de rôle déclinent leur prénom et leur nom. Le public découvre l'identité de celles et ceux qu'il va voir durant le spectacle, ils ne sont plus anonymes.

Puis, plusieurs séquences s'alternent : des prises de parole individuelles, debout, où chacun raconte un souvenir personnel qui prend place dans une thématique développée par séries. Ces séquences de prises de parole sont entrecoupées de moments collectifs, à l'avant-scène. Les mouvements d'ensemble permettent de laisser respirer la parole, de la faire résonner... mais ils donnent aussi à voir les corps, dans une chorégraphie minimaliste qui complète le travail sur la parole.

L'avant-dernière séquence du spectacle renvoie au «Je pense à vous » du titre. Je leur demande d'écrire une liste de noms en réponse à la question «À qui pensezvous après toutes ces années de vie ? ». Ils viennent se placer à tour de rôle sur la ligne de présentation et prononcent cette liste de noms, sans aucun autre détail. Ce moment mystérieux renvoie le participant à lui-même et à ses aimés, vivants ou disparus. A la fin, ils rejoindront leurs chaises et se retournent face à l'écran derrière eux, sur lequel sont projetées des photos en noir et blanc (elles sont toujours plus belles !) que je leur ai demandé d'apporter.

Je sélectionne les participants sur petite annonce dans des formes qui sont restées presque inchangées depuis l'origine: «Metteur en scène cherche hommes et femmes de plus de 70 ans pour travail professionnel sur le thème de la mémoire ». L'autre condition que je pose, outre à l'âge minimum, est depuis 2000 l'inexpérience totale dans le théâtre. En effet, pour ce projet j'avais besoin d'explorer une autre voie, celle du théâtre primitif, d'un retour aux sources en 
quelque sorte, où l' ' acteur », celui qui intervient, ne ferait pas appel à une expertise en la matière pour apparaitre nu et vulnérable.

Marina Repezza : Y a-t-il d'autres critères pour choisir les participants? Faitesvous une sélection parmi ceux qui répondent? Choisissez-vous «les plus intéressants $»$ ?

Didier Ruiz : Non, il n'y a pas d'enquête préliminaire pour savoir s'ils sont intéressants ou pas.

Je fais toujours confiance au hasard, d'où le principe de la petite annonce : on jette une bouteille à la mer. Je me suis toujours interdit de faire des choix, en me disant que si les gens répondaient à la petite annonce, c'est qu'il y avait déjà une curiosité, une folie en eux qui méritait d'être écoutée.

De fait, il n'y a pas d'autre sélection que celle que demandent les deux critères d'âge et de profession : il ne faut pas que ce soient d'anciens comédiens, justement pour que ça puisse marcher. J'ai connu en effet une mauvaise expérience en prenant une personne qui faisait beaucoup de théâtre amateur, lors de la première édition de mon spectacle. Il était La célébrité du théâtre amateur local et avait un regard constant sur ce qu'il fallait faire ou ne pas faire. Il n'avait absolument aucune spontanéité. C'est avec lui que j'ai eu le plus de problèmes, et c'est suite à cette expérience-là que je me suis dit jamais plus.

À partir de là, les gens qui se présentent, je leur explique le projet en tête à tête, et leur demande : «Est-ce que vous choisissez de travailler avec moi? » et les gens me disent oui ou non. Et à partir du moment où ils me disent oui, c'est parti.

Parfois aussi, $\mathrm{j}$ 'interviens si on voit que les petites annonces ne suffisent pas, je vais dans des lieux où il $\mathrm{y}$ a beaucoup de vieux. Maisons de retraite ou associations de joueurs de cartes, par exemple. Dans les grandes villes, à Moscou par exemple, les choses se déroulaient plus de cette manière parce que il n'y avait pas de petite annonce ; parce que l'Institut français avait uniquement décidé de fonctionner par du réseau. Il y avait la grand-tante de la responsable de la médiathèque qui était venue, et celle-ci en a parlé à un autre copain, etc. S'il n'est pas nécessaire d'avoir des personnes qui ne se connaissent pas, il est important qu'ils n'arrivent pas en bande. Le hasard est absolument primordial dans la constitution de l'équipe.

Marina Repezza : Comment travaillez-vous avec ces non-professionnels ? Car sur scène ils sont étonnamment naturels. Enregistrez-vous les séances? Est-ce que vous aboutissez à un texte écrit que les personnes concernées relisent ?

Didier Ruiz : Je n'enregistre pas les séances, mais écris en trame. J'ai une excellente mémoire et parviens donc à mémoriser avec une grande précision la manière dont ces personnes âgées racontent les choses et ce qu'elles me racontent. Et je sais que quand cela me touche d'emblée, au premier jet, il y a des chances pour que ça paraisse dans le montage final. 
Ils apprennent cette trame qui contient les souvenirs que j'ai sélectionnés et un ordre bien précis de succession dans les moments de prise de parole. C'est la seule chose que je leur demande d'apprendre. Ils l'apprennent par cœur et ils ont beaucoup de mal. Parce qu'ils sont vieux, ils ont plus l'habitude d'apprendre, parce que c'est très abstrait se mettre debout, parler après ou avant telle personne... c'est très compliqué ça. Parfois ils y arrivent, parfois pas. Alors quand ils n'y arrivent pas, c'est très simple, je leur dis d'écrire sur un bout de papier. Parfois c'est drôle parce qu'ils ne veulent pas reconnaître qu'ils ont du mal à apprendre par cœur. Je leur dis qu'ils peuvent écrire des billets, mais ils ne veulent pas admettre qu'ils ont des réels problèmes de mémoire. Une fois une des participantes avait tout écrit sur son poignet...

Mais il n'y a pas du tout de texte. Durant la première phase, je les vois trois fois une heure et demie en entretien individuel. Comme ça, à une table, avec un bloc. «Est-ce que vous vous souvenez de votre berceuse ? », ça c'est la première question, c'est toujours la même depuis 1999. Souvent, les gens ne comprennent pas, ils me demandent : «Quoi ? De ma quoi ? », je leur dis « De votre berceuse, quand vous étiez petit. Est-ce qu'on vous chantait une berceuse ? ». Il y en qui répondent «Non pas du tout, on ne me chantait pas de berceuse », puis des fois ils disent «si, je m'en souviens », et je leur demande de m'en chanter un bout. Ça enclenche quelque chose, ça doit enclencher quelque chose.

Marina Repezza: Dans le rapport émotionnel qui s'établit entre ces participants en performance et vous, puis par là même avec le public, est-ce que ce sont les souvenirs les plus importants pour les personnes qui sont les plus « efficaces»?

Didier Ruiz: Non. Souvent ils me disent «moi, je voudrais vraiment raconter ça, c'est très important pour moi », et moi je leur dis «vous verrez », il arrive un moment à la fin de ces trois entretiens, où je leur propose une première sélection à partir de ce qu'ils m'ont raconté.

Je garde environ $30 \%$ de ce qui y est raconté ; puis dans une deuxième phase, celle de répétition, intervient mon assistant qui les écoute jouer alors qu'il ne les avait vus qu'au démarrage de l'opération. On va refaire un tri à ce moment-là pour ne garder peut-être que 2-3\% de ce qui a été raconté ou vu pendant les entretiens.

Parmi les sélections, ne figure pas souvent ce qui apparaissait au début comme «le plus important », parce que le plus important pour chacun d'entre eux n'est pas le plus important pour moi. Ce qui correspond à mon critère de choix passe par la question « est-ce que ça me touche ou pas ».

Alors que le passage autobiographique qui croise le moment historique peut paraître le plus important pour le narrateur, cela peut ne présenter aucun intérêt, s’il ne provoque aucune émotion, soit dans le récit de la personne âgée, soit dans ce que ça représente. Dans la dernière édition, par exemple, il y avait un copain d'enfance de Guy Môquet, qui a été dans son réseau de résistance et a été arrêté un peu après lui. 
Môquet a été exécuté, et lui est resté en prison, il a fait toute une série de camps de prisonniers, jusqu'à la Libération. Et il voulait absolument parler de ça, parce qu'il s'agit d'un monsieur très respectable parce que résistant, militant, maintes fois médaillé. Mais pour moi, ce n'était pas ça le plus important. Il était passé de tel camp à tel camp, mais il n'y avait rien de très précis. Et je ne suis pas historien, je ne travaille pas sur l'internement de telle époque à telle époque !

Ce qui m'intéresse aurait été par exemple : dans cette forteresse, est-ce qu'à un moment donné il avait un souvenir de ses relations avec le gardien, ou avec un autre prisonnier ? Quelque chose de précis, de concret qui lui soit arrivé ? Et comme ce n'était pas le cas, toute cette période-là, je ne l'ai pas gardée.

Ce que j'ai gardé en revanche, c'est la rencontre qu'il avait eue avec ce petit garçon, puisqu'ils se sont rencontrés petits, quand ses parents étaient militants communistes, et un soir, en allant à une réunion de parti chez des gens, ils l'ont amené pour ne pas le laisser seul. Il se trouve que ce soir-là la réunion s'est tenue chez Monsieur et Madame Môquet, qui avaient un fils. Qui s'appelait Guy. Et c'est en arrivant chez M. et Mme Môquet que ses parents lui ont dit, «Voilà, ça c'est Guy, tu ne veux pas aller jouer avec lui ? », et il est allé jouer avec lui, et ils sont devenus très copains. Et cette amitié a duré toute une vie, jusqu'à l'exécution de Guy Môquet. Mais pour moi, c'était ça qui était plus important, plutôt que la suite d'une longue détention, qui n'était pas rattachée à quelque chose de très précis.

Le truc qu'on veut absolument caser est rarement intéressant, c'est pourquoi il faut prendre le temps d'écouter beaucoup. Et parfois trouver autre chose, d'absolument insignifiant, tout petit, mais qui a beaucoup plus de valeur, parce que beaucoup plus chargé. Encore une fois, je le redis peut-être, mon critère est : si ça me touche moi, il $\mathrm{y}$ a des chances que ça touche les gens. C'est par rapport à un souvenir, mais ensuite il y a l'écho que produisent les souvenirs mis côte à côte, des uns et des autres, et là est le travail des répétitions collectives, de mettre côte à côte des choses qui se font écho - ou pas. Et c'est l'ensemble, la mosaïque, qui produit, me semble-t-il, une émotion chez le public, parce que du coup les souvenirs ne sont plus forcément reliés à une seule personne, mais ils passent du côté du public, ils passent dans le collectif.

On passe de l'intime, extrêmement intime, de celui qui le livre, qui le donne avec ses mots, des mots souvent balbutiants... de cet intime on passe à du collectif, pur, parce qu'associé à une construction en «mosaïque », où les choses sont assemblées les unes à côté des autres, sans commentaire, et sans explication, ce qui est assez difficile à faire comprendre, parce que les gens veulent absolument expliquer : «oui, mais il faut que j'explique pourquoi mes parents étaient communistes. », «Mais non, je dis, ça c'est une autre histoire ! Moi ce qui m'intéresse c'est quand vous avez sonné à la porte de Monsieur et Madame Môquet, et qu'on vous a présenté un petit garçon qui avait votre âge ». 
En tout cas, certaines thématiques sont constantes, comme les souvenirs d'odeurs, les souvenirs de la première rencontre amoureuse et d'autres, ou encore les souvenirs de voyage, ceux liés au père...

Marina Repezza: Vous avez réalisé des éditions de «Dale Recuerdos » dans d'autres pays, notamment au Chili et en Russie, où vous avez rencontré un succès inouï. Comment cela s'est-il passé ? Est-ce que cela change votre manière de travailler que les participants ne parlent pas votre langue?

Didier Ruiz: Non, cela ne change pas du tout le principe. Du tout, très étrangement. La forme du travail était exactement la même au Chili où je me suis interdit de me documenter sur tel ou tel évènement. Et quand j'y suis arrivé, je connaissais peu de l'histoire de ce qu'avaient vécu les gens. Et c'est en laissant faire que des choses très fortes sont apparues, à Santiago, par exemple.

On se rend compte très vite de la dictature sanglante qu'a connu le pays et à quel point le coup d'état du 11 septembre 1973 est une date clé de son histoire. Et je me suis dit, tiens, je vais leur poser la question. Savoir ce qu'ils faisaient ce jour-là. Et la première en entretien à qui je demande ça, je la vois changer de visage. Et elle me dit «bien sûr, je me souviens très bien de ce que je faisais ce jour-là, comment voulez-vous que j'oublie ». Elle me décrit absolument tout son planning de la journée, du matin au soir (elle était à la fenêtre, elle nettoyait les légumes à l'évier, elle avait des affaires à repasser, etc.) Tout, tout, tout, très précisément.

Je voyais bien qu'ils se mettaient à raconter dans une société qui veut aujourd'hui occulter ces évènements-là (pour la jeunesse chilienne, Allende et Pinochet... c'est vraiment le Moyen-Âge !).

Il y a des choses très belles qui se sont passés à Santiago puisque parfois les gens ne voulaient pas parler... Les gens disaient : «Mais moi je ne peux pas dire ça, je ne l'ai jamais dit à personne !» «Attendez, Pinochet il est mort et la dictature, c'est fini ». « Oui, mais quand même... Vous croyez, Didier, que je vais dire ça ? ».

Dans toutes nos performances de par le monde, on a toujours gardé le même nombre de représentations qu'on a limitées à trois. Durant la seconde année d'expérience avec le projet «Dale Recuerdos. Je pense à vous », on a limité à trois car on s'est rendu compte qu'à la quatrième représentation se produisait un phénomène d'habitude, et de cabotinage.

Mais il y a eu l'exception russe, qui a été repris quatre fois sur quatre ans. Ça a été un tel succès à Moscou que ça a été repris l'année suivante, puis un programmateur de spectacle lyonnais était venu à Moscou et a voulu les faire venir à Lyon, et enfin, l'année d'après, une tournée en Russie centrale. On a donc à chaque fois re-répété. Mais ces Russes, ils étaient très pro, parce qu'à aucun moment il n’y a eu d'altération de la fraîcheur, pour des raisons que je ne saurais expliquer. À Moscou, ça a eu un succès... inimaginable, ce spectacle. J'ai eu ce prix extrêmement prestigieux de l'Académie de Théâtre russe. Toute la presse en a parlé, c'était incroyable. 
Mais je crois que ça venait de leur humilité à eux. Il y avait quelque chose de très soviétique dans la participation de ces personnes âgées, de très soigneux et «au service de... », au service d'un projet.

Ils avaient été tellement bouleversés par les retours ! Les spectateurs debout, en larmes, qui leur jetaient des bouquets de fleurs. C'était hallucinant ! Le public avait énormément besoin d'entendre leur histoire, d'entendre tous ces vieux qui étaient du coup leur grand-père et leur grand-mère... Entendre cette histoire terrible, car sur les dix participants, il y en avait les trois-quarts qui avaient perdu des proches de leur famille ou pendant la guerre, ou avant la guerre dans les famines, ou après, dans les purges : dans les camps ou dans les goulags... Et du coup, le public - jeune - entendait une histoire, tandis que l'environnement quotidien laisse à penser qu'ils veulent oublier. Si tu vas à Moscou aujourd'hui, tu as l'impression que c'est comme à NewYork, que tout le monde est dans l'opulence. Tout relevait d'une importance capitale, dans ces représentations : les appartements communautaires notamment, d'où ces gens étaient issus, car ils avaient tous vécus dans des appartements communautaires.

Il y avait une femme extraordinaire, que j'aimais beaucoup, qui s'appelait Ludmilla. Sa maman était médecin. Pendant la guerre elle habitait dans un immeuble. Tous les voisins avaient brûlé les livres, parce qu'il n'y avait plus de chauffage, il y avait rien, c'était terrifiant. Tout le monde avait brûlé les livres pour se chauffer. Et eux, ils avaient refusé de brûler les livres. Il y avait donc une bibliothèque dans cet appartement, qui servait de bibliothèque à tout l'immeuble, et les livres étaient tellement usés à force d'être prêtés, rendus, utilisés, que Ludmilla petite fille était employée à réparer les livres. Et sa maman, médecin, lui disait : «maintenant on va opérer les livres, on va les guérir », en faisant coller du scotch à sa petite fille. Et, véritable hasard de l'histoire, plus tard, cette Ludmilla est devenue bibliothécaire à la bibliothèque de Moscou.

Marina Repezza : Revenons à un point de vue plus général sur votre projet scénique : j'ai entendu un de vos participants parler du caractère thérapeutique de votre travail. Est-ce que cela influe sur votre relation avec eux ?

Didier Ruiz : En fait, je ne les interroge jamais sur pourquoi ils sont venus. Je ne pose jamais cette question : «pourquoi êtes-vous là ? ». Et ceci dit, ils ne me le disent jamais. Non plus. Donc ce qui se passe après, j'ai envie de le dire, ne m'intéresse pas, très égoïstement. En même temps ce n'est pas vrai... puisqu'on s'écrit. Mais on ne s'écrit pas pour qu'ils me disent, ou pour que j'entende «vous avez changé ma vie... ». On s'écrit, justement, pour garder une trace.

Marina Repezza : Vous répondez à tous vos participants ? Il doit y avoir beaucoup de gens maintenant... 
Didier Ruiz : Oui, beaucoup. Oui, je leur écris pour les vœux, donc j'en envoie, cent-soixante... à peu près. Et je reçois beaucoup de courrier, assez bouleversant. Dans le courrier de ce matin il doit y en avoir. Tiens, ça c'en est une.

Marina Repezza : Presque tous les jours, donc ?

Didier Ruiz : À cette époque de l'année oui. (Il lit un extrait de la lettre qu'il a reçue le matin même.) « Merci de ne pas nous oublier, c'est un vrai réconfort dans ce monde de brutes. Je me permets de vous embrasser. » Oui, c'est l'histoire de l'oubli qui est toujours là... L'oubli, c'est la pire des choses !

Marina Repezza : Être oublié ou oublier?

Didier Ruiz : Les deux. Être oublié c'est atroce. Et oublier c'est impardonnable. Les gens qui te disent : «Oh, je t'ai oublié ! »... Être oublié c'est être nié, c'est déjà être mort avant la mort ! Une mort avant la mort qui est insupportable.

Marina Repezza : Liez-vous la mémoire à la mort ?

Didier Ruiz : Oui, pour moi la mémoire fait partie de la trace, c'est à dire de ce qui reste. Je me demande comment, jusqu'à quel point les choses restent. Ce qui est terrifiant c'est que, à un moment donné tu pioches dans un album photo, tu vois une photo et tu ne sais plus qui sont ces gens autour de toi. Plus personne n'est là pour te le dire, et ce qui était connu devient invisible, inconnu. C'est le cas pour moi, dans mes photos de famille. Mes oncles, mes tantes et mes parents sont morts. On se retrouve alors à avoir des photos où l'on ne sait plus qui c'est.

Et cette idée de la trace, de suivre la trace jusqu'au moment inscrit sur une ligne, où l'on passe, presque à la seconde près, de ce monde visible à l'invisible... Ce moment-là m'interroge toujours. Est-il est possible d'agir sur ce curseur ? Peut-on le déplacer, faire en sorte de se souvenir un tout petit peu plus longtemps ? Est-ce qu'il est possible de le faire disparaître ? Ou de se souvenir toujours de tout ? (Chose impossible). Comment fonctionne alors cette mémoire, est-ce qu'on est obligé de vider pour remettre ? Et surtout, par rapport à la mort, qu'est-ce qui reste au moment où l'on va partir... Qu'est-ce qui reste dans cette mémoire, qu'est-ce qui reste dans cette valise, de quoi tu te souviens, au bout du compte, quels sont les dix éléments qui te restent, quand tu es au bout du chemin, où tu as accumulé des tonnes d'informations, des tonnes de visages, des tonnes de noms, des tonnes d'émotions... Quand tu arrives à la fin, il te reste peut-être dix, quinze, vingt souvenirs, dans une toute petite valise. Lesquels, et pourquoi ? Je trouve ça absolument passionnant. 